\title{
Nuclear Resonance Fluorescence for Materials Assay
}

\author{
Brian Quiter, Bernhard Ludewigt, Vladimir Mozin, Stanley G. Prussin
}

\begin{abstract}
This paper discusses the use of nuclear resonance fluorescence (NRF) techniques for the isotopic and quantitative assaying of radioactive material. Potential applications include age-dating of an unknown radioactive source, pre- and postdetonation nuclear forensics, and safeguards for nuclear fuel cycles Examples of age-dating a strong radioactive source and assaying a spent fuel pin are discussed. The modeling work has ben performed with the Monte Carlo radiation transport computer code MCNPX, and the capability to simulate NRF has bee added to the code. Discussed are the limitations in MCNPX's photon transport physics for accurately describing photon scattering processes that are important contributions to the background and impact the applicability of the NRF assay technique.
\end{abstract}

Index Terms-Nuclear Safeguards, non-destructive analysis, nuclear resonance fluorescence, MCNPX.

\section{INTRODUCTION}

$\mathrm{N}$ UCLEAR Resonance Fluorescence (NRF) has been a known physics phenomenon for over 40 years [1]. Initial interest was devoted to nuclear structure studies. More recently, NRF has been identified as a promising technology for the assaying of materials. In the past several years, the US government has funded cross section measurements for ${ }^{239} \mathrm{Pu}$ and ${ }^{235} \mathrm{U}$ for potential application to the screening of cargo for nuclear weapons materials [2],[3].

NRF is an attractive non-destructive analysis (NDA) method because NRF signatures are unique identifiers of a nuclide within a sample. Beyond the cargo screening problem, there are other applications where knowledge of the isotopic composition of a material is important: determining the time since chemical separation of a radiological source, nuclear safeguards including measuring uranium enrichments, spent fuel compositions, waste and product materials and confirming shipper/receiver declarations, and nuclear forensics.

Manuscript received June 29, 2009. This work is supported by an ARI grant from the National Science Foundation and the Department of Homeland Security BS123456 and by the Director, Office of Science of the US Department of Energy at Lawrence Berkeley National Laboratory under contract number DE-AC02-05CH11231.

B. Quiter, S. Prussin, and V. Mozin are with the Nuclear Engineering Department of the University of California, Berkeley, Berkeley, CA 94720 USA (510-642-5274; fax: 510-643-9685; e-mail: bquiter@ berkeley.edu).

B. Ludewigt is a scientist at the Lawrence Berkeley National Laboratory, Berkeley, CA 94720 USA.
Destructive chemical analysis is the assay method currently applied to many of these applications

Determining the time since chemical separation of a radiological source is presently done by analyzing a sample taken from radiological sources to determine relative concentrations of parent and daughter isotopes. However, older radioactive sources may undergo chemical speciation when radioactive decay causes chemical changes. This makes sampling problematic, and a non-destructive analysis alternative to dissolution of the entire sample is desirable.

Possibilities and limitations of NRF-based assays for both, spent fuel and age-dating of large sealed-source radiators are discussed below.

\section{NUCLEAR RESONANCE FLUORESCENCE}

\section{A. Physics}

NRF is the process in which a nucleus absorbs a photon and is excited to a specific excited state that subsequently deexcites by emission of one or more $\gamma$-rays. By measuring the energies of gamma rays emitted during de-excitation of an NRF state within a medium, one can uniquely identify the presence of the isotope whose state was observed. If the assay geometry and the strength of a measured NRF transition are known, the intensity of a measured NRF signal can be used to quantify the concentration of the isotope within a material. The physics and measurement of NRF has been discussed extensively elsewhere [1].

The energies associated with NRF transitions are analogous to the energy ranges of excited nuclear states; i.e. few $\mathrm{keV}$ to many $\mathrm{MeV}$. However, to use NRF for NDA, we require photons capable of penetrating through macroscopic quantities of material. To keep background photon rates as low as is practical, it is preferable to avoid the production of photoneutrons. We therefore restrict our attention to NRF levels in the range of approximately 1.5 to $4 \mathrm{MeV}$, where photons are maximally penetrating, yet below particleemission threshold of most isotopes.

\section{B. Non-destructive Analysis Methodology}

The NDA method proposed here is quite simple. A bremsstrahlung beam, generated by impinging electrons upon a thick, high $\mathrm{Z}$ converter such as tungsten, is collimated and directed onto the target to be assayed. Photons at resonant 
energies excite NRF states and the subsequent $\gamma$-rays, emitted during de-excitation, are detected by high purity germanium (HPGe) detectors located at backward-angles relative to the incident beam direction. A schematic of the system geometry is shown in figure 1.

In this study the photon fluence at an assumed detector location due to all processes that contribute to the fluence has been evaluated to determine whether NRF signals from materials of interest could be identified above background contributions. Radioactive emission from the target is independent of the interrogating beam and is treated separately from the background intensity due to scattering of bremsstrahlung photons. The radioactivity of the target defines the minimum necessary flux of bremsstrahlung photons needed to generate an NRF signal larger than that background. Shielding between the assayed target and the detector is needed to suppress the flux of lower energy photons and allow the detector to operate without prohibitive pulse pile-up.

The interrogating photon spectrum is computed by simulating in MCNPX monoenergetic electrons impinging upon a bremsstrahlung converter and tallying the flux of emitted photons into discrete angular bins[9]. The photon beam is then transported from the converter through the target using the geometry shown in figure 1 to determine the resulting photon fluence at the detector location.

The fluence is calculated for the resonant nuclear scattering (NRF) and for the non-resonant background and these values are compared. The non-elastic scattering contribution is simulated with MCNPX. However, non-resonant

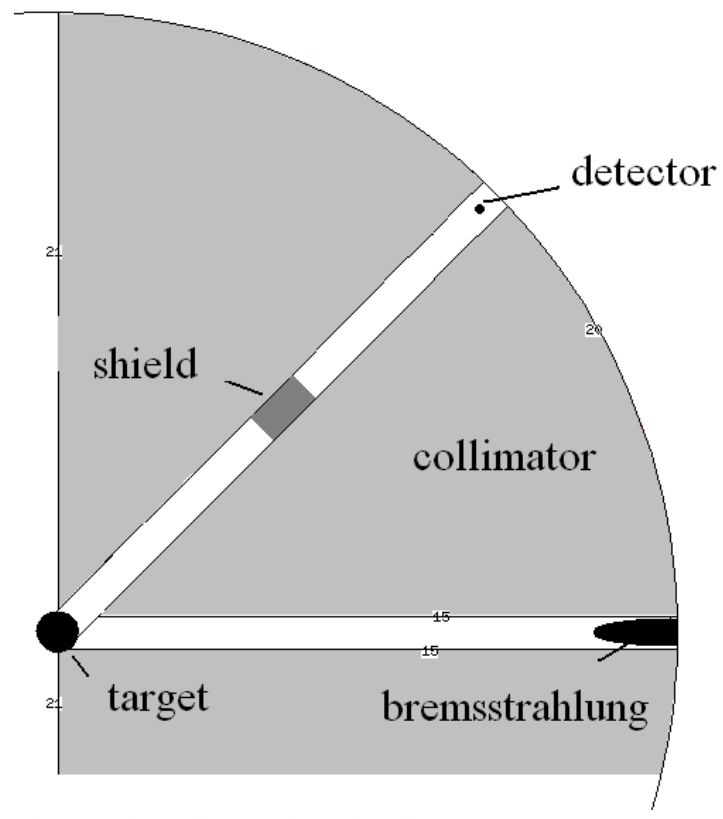

Fig. 1. Schematic overview of studied NRF assay geometry.

photonuclear processes such as Rayleigh, Thompson, and Delbruck scattering are not calculated by MCNPX. Furthermore, MCNPX only considers Rayleigh scattering for a limited angular range of photon emission. Rayleigh scattering is not simulated at all for momentum transfers between the photon and electron is more than $6 \mathrm{~A}^{-1}$ of electron momentum. For the geometry studied, these elastic scattering processes result in a significant contribution to the background photon spectrum and thus were calculated separately. Table 1 lists the maximum scattering angle simulated by Rayleigh scattering in MCNPX for relevant photon energies.

Coherently scattered photons to the background spectrum, interrogating bremsstrahlung spectra have been folded with elastic scattering cross sections tabulated in the RTAB database [10] and geometrically attenuated. These spectra are labeled due to elastic scattering in the following figures.

TABLE I

MAXIMUM RELATIVE SCATTERING ANGLE COMPUTED BY MCNPX FOR RAYLEIGH SCATTERING OF PHOTONS

\begin{tabular}{|l|l|}
\hline \multicolumn{2}{|c|}{ RAYLEIGH SCATTERING OF PHOTONS } \\
\hline $\mathrm{E}(\mathrm{MeV})$ & Angle (degrees) \\
\hline 0.1 & 96 \\
\hline 1.73 & 5 \\
\hline 3.76 & 2.25 \\
\hline
\end{tabular}

Any NRF assaying technique requires that the NRF signal must be detectable against the background photons created by both, the assaying photon spectrum and radioactive decay. While increasing the photon source's intensity will increase the intensity of the NRF signal relative to a radioactive background, it will not improve NRF signal strength relative to the scattered interrogation photon intensity.

\section{SPECIFIC EXAMPLES}

\section{A. Spent Nuclear Fuel}

A critical component of any nuclear safeguards methodology is the assurance that nuclear materials generated during peaceful nuclear activities are not diverted to weapons fabrication. At fuel reprocessing facilities, the quantity of $\mathrm{Pu}$ entering and leaving the reprocessing scheme is measured to ensure no diversion has occurred. In aqueous reprocessing facilities, the measurement of $\mathrm{Pu}$ and $\mathrm{U}$ concentration in spent fuel is determined by chemical analysis after the fuel has been dissolved for reprocessing. With proposed non-aqueous reprocessing techniques, new assay methods are needed to measure Pu content in spent fuel without aqueous dissolution. In this light, using NRF to perform NDA on spent fuel has been considered [11]. 


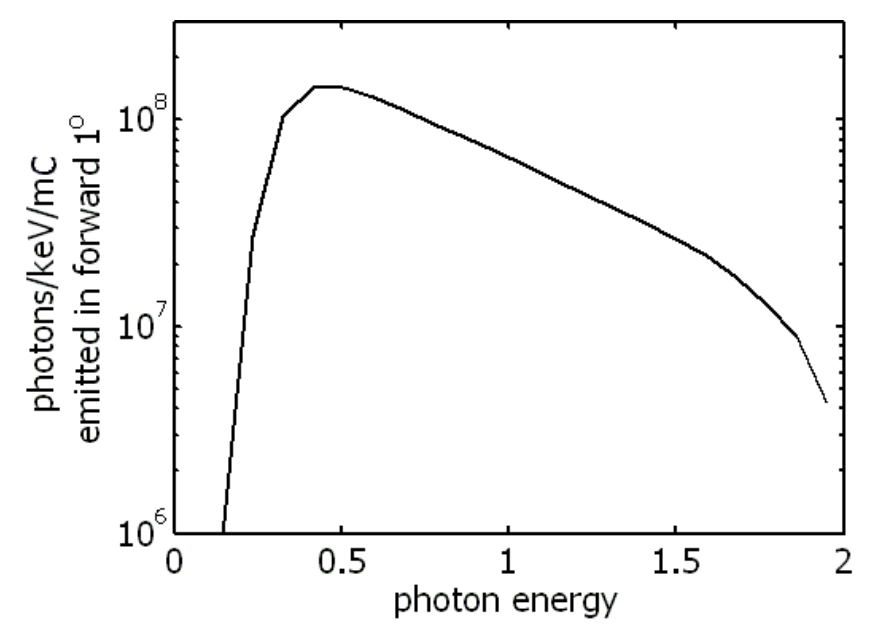

Fig. 2. Calculated bremsstrahlung spectrum emitted in forward 1 degree when $2 \mathrm{MeV}$ electrons impinge upon a $4.5 \mathrm{~mm}$ thick tungsten slab.

The radioactive background due to spent fuel activity is the first limit that must be considered. A $38 \mathrm{GWD} / \mathrm{MTU}$ burnup BWR fuel pin with a 12 year cooling time was considered. The composition was calculated by the code Origen in the Scale 5.1 software package [12]. The fuel pin comprised of $0.41 \%{ }^{235} \mathrm{U}$ by mass. In the geometry considered (see below) the photon fluence due to radioactive decay has been computed at the assumed detector location (see figure 1). The spectrum has a drop-off of 3 orders of magnitude above 1.6 $\mathrm{MeV}$ where ${ }^{154} \mathrm{Eu}(8.59 \mathrm{yr})$ has a strong line. NRF lines must be higher in energy than the ${ }^{154} \mathrm{Eu}$ line to be visible. The overall intensity of the radioactive decay spectrum is dominated by lower energy photons. Placing approximately 1 " of tungsten between the fuel pin and the detector reduces the photon intensity to an acceptable level.

NRF states have not yet been thoroughly mapped for all actinides. So far NRF responses of ${ }^{235} \mathrm{U},{ }^{239} \mathrm{Pu}[4],[5],{ }^{238} \mathrm{U}$ and ${ }^{236} \mathrm{U}$ have been measured [18],[19] and an experiment to measure the NRF response of ${ }^{237} \mathrm{~Np}$ has recently been conducted [7]. The strongest ${ }^{235} \mathrm{U}$ resonance measured is at $1.733 \mathrm{MeV}$ with a cross section of $30 \mathrm{eV} \cdot \mathrm{b}$. Interrogating this resonance with a $2 \mathrm{MeV}$ endpoint energy bremsstrahlung spectrum provides nearly maximal signal intensity relative to the scattered photon intensity. The simulated $2 \mathrm{MeV}$ endpoint energy bremsstrahlung photon spectrum for photons emitted from a $4.5 \mathrm{~mm}$ thick tungsten converter within the forward 1 degree of the incident electron beam direction is shown in figure 2. This spectrum is transported in the interrogation geometry seen in figure 1 . The converter and detector are both located $45 \mathrm{~cm}$ from the $1 \mathrm{~cm}$ diameter spent fuel pin within a 1 $\mathrm{cm}$ diameter cylindrical viewing windows in a spherical tungsten collimator. Bremsstrahlung photons emitted within the collimator dimension are transported to the fuel pin, whereas photons leaving the converter outside of the collimator dimension have been determined to be sufficiently attenuated by $45 \mathrm{~cm}$ of tungsten that they can be neglected. Photon fluences have been calculated at the point labeled 'detector location' and fluences due to the three mechanisms discussed with no tungsten shield above are shown in figure 3 . The discrete peaks indicating ${ }^{235} \mathrm{U}$ NRF photons are placed in $3 \mathrm{keV}$ wide bins to simulate the effective energy resolution of an HPGe detector. These peaks exceed the scattered spectrum as computed by MCNPX, however, the fluence due to elastic scattering mechanisms (not in MCNPX) is significantly higher than the signal. This indicates that the directly measured NRF signal from actinides in spent fuel at backwards angles may only be sufficiently strong for constituents with concentrations greater than roughly $5 \%$.

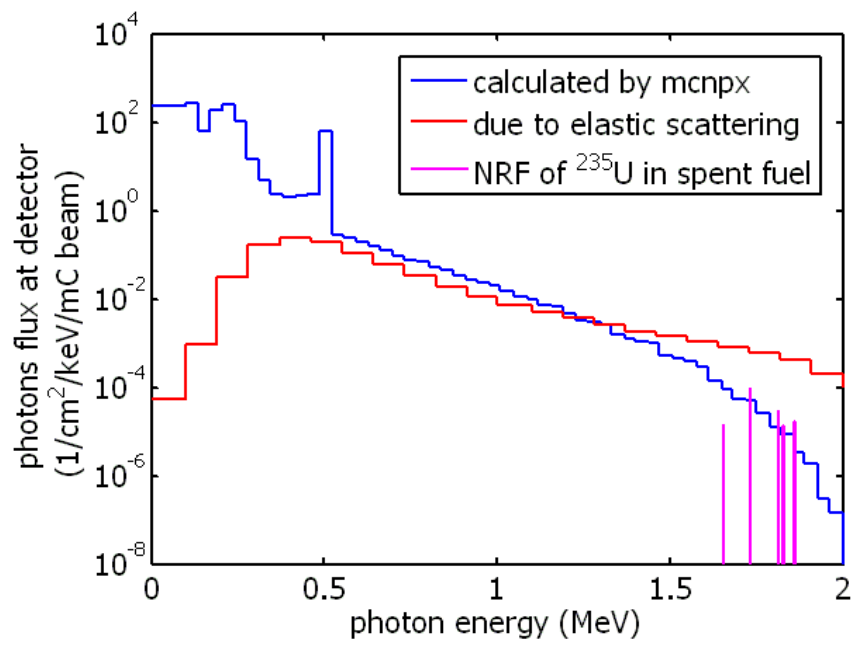

Fig. 3. Calculated contributions to photon spectrum at detector location for assay of a spent fuel pin containing $0.41 \%{ }^{235} \mathrm{U}$. In this example no shielding is present between the target and the detector location.

\section{B. Industrial Radiator}

The possible use of large radioactive sources in radiation dispersal devices (RDDs) is of considerable national and international concern. Of particular concern are the longerlived fission product isotopes ${ }^{90} \mathrm{Sr}$ and ${ }^{137} \mathrm{Cs}$ that exist in sources whose activities can exceed $10^{4} \mathrm{Ci}$. The age of such sources, i.e. the age since discharge of the fuel sample from the reactor and the age since chemical isolation of the radioisotope from the fission products, have been identified as important parameters for forensic purposes, especially if other identifying characteristics are missing or uncertain. Sources used in the public and private domains can have ages up to 60 $\mathrm{y}$.

Although such age dating can, in principle, be accomplished with high accuracy and precision by careful use of radiochemical and mass spectrometic methodologies, there are significant shortcomings in practice. Of primary importance is that essentially all quantitative radiochemical methods require wet chemical manipulations on a representative sample of the entire source, and thus the source must be opened and processed. For sources that have decayed for a time greater than one half-life, stoichiometric changes coupled with thermal effects can cause significant segregation of elements, making isolation of $\quad$ a $\quad$ representative 


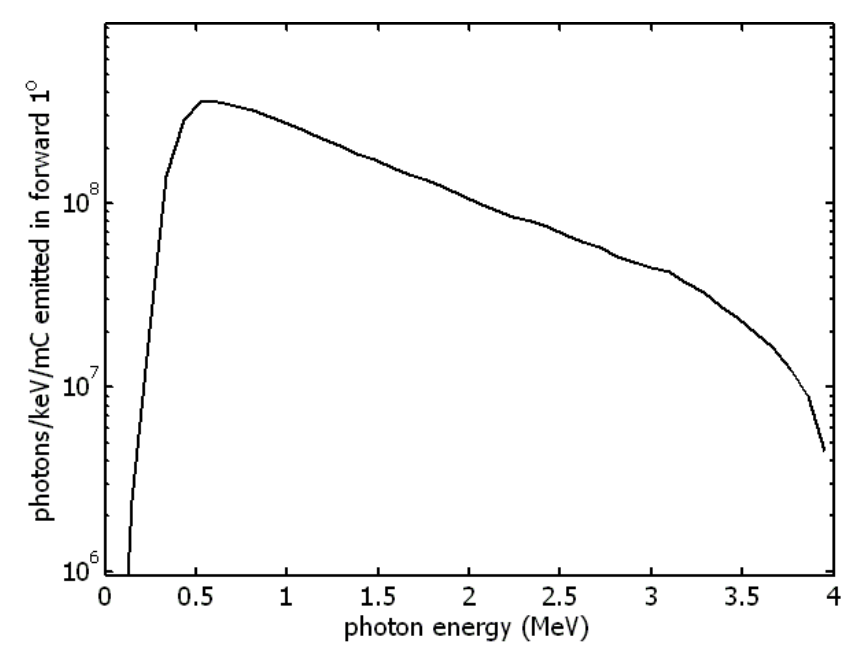

Fig. 4. Calculated bremsstrahlung spectrum emitted in forward 1 degree when $4 \mathrm{MeV}$ electrons impinge upon a $7.5 \mathrm{~mm}$ thick tungsten slab.

sample difficult without dissolving and homogenizing the entire source. While this is straightforward with weak sources, sampling more than $10^{2} \mathrm{Ci}$ in such a manner is a daunting task. Second, there are only few facilities that can handle such large sources and the disposal of the predominant bulk of the radioactivity from an opened source as well as decontamination of equipment is both difficult and expensive. Thus performing NDA on industrial irradiators via NRF is an attractive method to age-date a recovered source. A $10^{3} \mathrm{Ci}$ ${ }^{137} \mathrm{Cs}$ source is considered as an example.

The NRF spectrum of ${ }^{137} \mathrm{Cs}$ has not been measured, however the NRF response of its daughter, ${ }^{137} \mathrm{Ba}$ has been measured [15] and NRF-based analysis for ${ }^{137} \mathrm{Ba}$ alone can provide significant information for age-dating. The ${ }^{137} \mathrm{Xe} \beta$ - decay has been studied and several excited states of ${ }^{137} \mathrm{Cs}$ between 3.5 and $4 \mathrm{MeV}$ that decay directly to the ground state have been reported [8]. It is probable that these states will exhibit an NRF response as well. We estimate the NRF response of the $3.761 \mathrm{MeV}{ }^{137} \mathrm{Ba}$ resonance and if the ${ }^{137} \mathrm{Cs}$ NRF response is of similar intensity, we expect our conclusion to be valid for measuring ${ }^{137} \mathrm{Cs}$ via NRF as well.

${ }^{137} \mathrm{Cs}$ sources are generally produced by chemically separating cesium from fission products which contain ${ }^{133} \mathrm{Cs}$, ${ }^{135} \mathrm{Cs}$, and ${ }^{137} \mathrm{Cs}$. These have fission yields of $6.7 \%, 6.54 \%$, and $6.19 \%$ respectively, quite independent of reactor type. This implies that the Cs in a ${ }^{137} \mathrm{Cs}$ source will initially be about $31.9 \%{ }^{137} \mathrm{Cs}$. Cs in irradiators is typically in the chemical form $\mathrm{CsCl}$, and the sealed containment tends to be a steel alloy of a few mm thickness [16].

The assay geometry is identical to that shown in figure 1, except that the target is now a sphere of $\mathrm{CsCl}$ in a $3 \mathrm{~mm}$ thick steel shell, and that the bremsstrahlung beam used for interrogation (figure 4) was produced by impinging $4 \mathrm{MeV}$ electrons upon a $7.5 \mathrm{~mm}$ thick tungsten converter. The detector viewing a source from a distance of $45 \mathrm{~cm}$ will require approximately $13.2 \mathrm{~cm}$ of lead shielding to reduce the count rate of the $661 \mathrm{keV} \gamma$-ray to acceptable levels, which for this example we have conservatively limited to $10^{4} \mathrm{cps}$ [17].

The three different contributions to the photon fluences at the detector location calculated as discussed above are shown in figure 5. With $13.2 \mathrm{~cm}$ of lead shielding the count rate due to NRF in a single $90 \%$ relative efficiency HPGe detector is estimated to be approximately 2 counts per coulomb of electrons incident upon the bremsstrahlung converter. The total photon fluence due to scattering of bremsstrahlung photons would be approximately 500 photons $/ \mathrm{cm}^{2} /$ milicoulomb, resulting in low detector count rates. The NRF signal is calculated to be approximately 10 times as intense as the non-NRF photons for an energy bin of 4 $\mathrm{keV}$ width that takes into account the HPGe detector's energy resolution at $3.76 \mathrm{MeV}$. With a $100 \mu \mathrm{A}$ beam and six HPGe detectors (90\% relative efficiency), collecting $10{ }^{3}{ }^{137} \mathrm{Ba}$ NRF counts from a source where the majority of the ${ }^{137} \mathrm{Cs}$ had already decayed would require approximately 24 hours of assay.

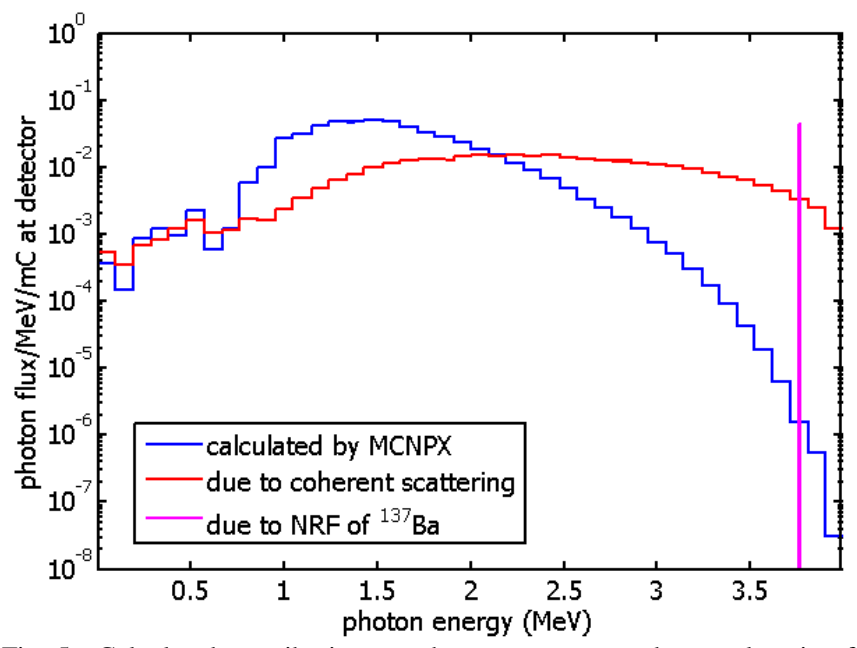

Fig. 5. Calculated contributions to photon spectrum at detector location for assay of a $1 \mathrm{kCi}$ initial activity $\mathrm{CsCl}$ irradiator after ${ }^{137} \mathrm{Cs}$ has completely decayed.

\section{CONCUSIONS}

Assaying materials by directly measuring bremsstrahlunginduced NRF lines has been studied. The radiation transport code MCNPX was used to simulate the non-resonant background but the code includes only a truncated treatment of atomic Rayleigh scattering and misses photo-nuclear physics. Scattering mechanisms not simulated in MCNPX are major contributions to the scattered photon background at the backward angles for photon energies of interest. Addition of these processes to the scattered photon intensity as calculated by MCNPX indicates that the total non-resonant photon background may not allow the assaying of $\mathrm{Pu}$ and ${ }^{235} \mathrm{U}$ concentrations in typical spent fuel by the method described here.

The sensitivity of the method can, in principle, be proportionally improved by improving the energy resolution of 
the detector. The use of a foil of nearly isotopically pure material, a "witness" foil, as a proxy for a detector may alleviate the problem. This method has been previously proposed for cargo interrogation [13] and is now the subject of further investigation. Likewise, decreasing the bandwidth of the interrogating photon beam could improve sensitivity, but the measured elastic scattering background component only improves once the bandwidth becomes smaller than the detector's resolution. Thus, even Compton-backscatter photon sources [14] under development for other NRF applications are not expected to significantly improve the sensitivity of the method.

The method of direct measurement of NRF photons does appear to be sufficiently sensitive to measure the concentration of ${ }^{137} \mathrm{Ba}$ in a large ${ }^{137} \mathrm{Cs}$ source after it has decayed for approximately 5 years. If the $\mathrm{NRF}$ response of ${ }^{137} \mathrm{Cs}$ is comparable to that of ${ }^{137} \mathrm{Ba}$, the method will also be sensitive to the ${ }^{137} \mathrm{Cs}$ concentration, thus relative concentrations of ${ }^{137} \mathrm{Cs}$ and ${ }^{137} \mathrm{Ba}$ may be measured and age-dating directly accomplished. Sufficient counting statistics can be accomplished within approximately 1 day of measurement for reasonable assay setups using available technology.

\section{REFERENCES}

[1] U. Kneissl et al. "Investigation of Nuclear Structure by Resonance Fluorescence Scattering," Prog. Part. Nucl. Phys. 37 1996, pp. 349.

[2] G. Warren et al. "Nuclear Resonance Fluorescence of 235U" IEEE Nuclear Science Symposium 2006, pp. 914.

[3] W. Bertozzi and R. Ledoux, "Nuclear resonance fluoresce imaging in non-intrusive cargo inspection," NIM B 241 2005, pp. 820.

[4] M. Johnson et al. "Final Task Report on NRF Measurements of Photon Scattering Resonances in Plutonium at the High Voltage Research Laboratory of MIT," UCRL-TR-228387 2007.

[5] W. Bertozzi et al. "Nuclear resonance fluorescence excitations near $2 \mathrm{MeV}$ in ${ }^{235} \mathrm{U}$ and ${ }^{239} \mathrm{Pu}$," Phys. Rev. C. 78, 0416012008.

[6] J. Pruet et al. "Detecting clandestine material with nuclear resonance fluorescence”. J. App. Phys. 99, 1231022006.

[7] E. Norman, UC Berkeley Dept. of Nuclear Engineering, private communication, May 2009.

[8] W.R.Western et al. Decay of Mass-Separated ${ }^{137}$ Xe to Levels in the $N=82$ Nucleus ${ }^{137}$ Cs. Phys. Rev. C 15, 1977, pp. 1024.

[9] D. Pelowitz MCNPX, LA-CP-07-1473, 2008.

[10] L. Kissel, Radiat. Phys. Chem. 59 2000, 185. Available: http://adg.llnl.gov/Research/scattering /RTAB.html

[11] S. Tobin et al. "Survey of Seven Measurement Techniques for Quantifying the Fissile Content of Spent Fuel" LA-UR-07-3336 (2007).

[12] SCALE: A Modular Code System for Performing Standardized Computer Analyses for Licensing Evaluations, ORNL/TM2005/39, Version 5.1, Vols. I-III, November 2006. Available from Radiation Safety Information Computational Center at Oak Ridge National Laboratory as CCC-732.

[13] W. Bertozzi, "Adaptive scanning of materials using nuclear resonance fluorescence imaging," US Patent 7120226, October 10, 2006.

[14] F. Hartemann et al. "Gamma-ray Compton Light Source Development at LLNL," Proceedings of PAC07, pp. 1245-1247.

[15] M. Scheck et al. "Dipole strength distributions in the stable Ba isotopes ${ }^{134-138} \mathrm{Ba}$ : A study in the mass region of a nuclear shape transition," Phys. Rev. C 70, 044319, 2004.
[16] Waste Encapsulation and Storage Facility Basis for Interim Operation, Fluor Hanford, Richland, WA, 2000. Document HNFSD-WM-BIO-02.

[17] R.M. Keyser et al. "Improved performance in germanium detector gamma-spectrometers

[18] J. Margraf et al. "Photoexcitations of low-lying dipole transitions in ${ }^{236}$ U," Phys. Rev. C 42 1990, pp. 771.

[19] A. Zilges et al. "Strong dipole excitations around 1.8 MeV in ${ }^{238}$ U," Phys. Rev. C 52 1995, pp. R468-470 